Article

\title{
Preparation of Poly(acrylic acid) Hydrogel by Radiation Crosslinking and Its Application for Mucoadhesives
}

\author{
Young-Chang Nho ${ }^{\dagger} *$, Jong-Seok Park ${ }^{\dagger} *$ and Youn-Mook Lim
}

Research Division for Industry and Environment, Korea Atomic Energy Research Institute, 29 Geumgu-gil, Jeongeup-si, Jeollabuk-do 580-185, Korea; E-Mail: ymlim@kaeri.re.kr

$\dagger$ These authors contributed equally to this work.

* Authors to whom correspondence should be addressed; E-Mails: jaspa@hanmial.net (Y.-C.N.); jspark75@kaeri.re.kr (J.-S.P.); Tel.: +82-63-570-3060 (Y.-C.N.); +82-63-570-3067 (J.-S.P.); Fax: +82-63-570-3069 (Y.-C.N.); +82-63-570-3079 (J.-S.P.).

Received: 8 January 2014; in revised form: 7 March 2014 / Accepted: 13 March 2014 /

Published: 19 March 2014

\begin{abstract}
A mucoadhesive drug delivery system can improve the effectiveness of a drug by maintaining the drug concentration and allowing targeting and localization of the drug at a specific site. Acrylic-based hydrogels have been used extensively as a mucoadhesive system owing to their flexibility and excellent bioadhesion. In this experiment, poly(acrylic acid) was selected to prepare the bioadhesive hydrogel adhering to mucosal surfaces using a radiation process. Poly(acrylic acid) was dissolved in water to a prepare poly(acrylic acid) solution, and the solution was then irradiated by an electron beam at up to $75 \mathrm{kGy}$ to make hydrogels. Their physical properties, such as gel percent, swelling percent and adhesive strength to mucosal surfaces, were investigated. Triamcinolone acetonide was used as a model drug. The dried poly(acrylic acid) film was dipped in a $0.1 \mathrm{wt} \%$ triamcinolone acetonide solution in ethanol, and then dried at $25{ }^{\circ} \mathrm{C}$. The release of triamcinolone acetonide was determined at different time intervals, and UV (Ultraviolet)-Vis spectroscopy was used to determine the released concentration of triamcinolone acetonide at $238 \mathrm{~nm}$. It was shown that poly(acrylic acid)-based drug carriers were successfully prepared for use in a bioadhesive drug delivery system.
\end{abstract}

Keywords: mucoadhesive; oral cavity; drug carriers; poly acrylic acid (PAA); electron beam; irradiation 


\section{Introduction}

Drug delivery systems have been used for reducing side effects, enhancing therapeutic efficacy and improving patient compliance. Many different kinds of polymers have been examined as potential carriers to deliver a drug in an efficient and controlled manner. A transmucosal drug delivery (TMD) system is one of many different methods used in drug delivery systems. A TMD system is applied on a mucous membrane and delivers a drug across the mucous membrane to achieve a local or systemic effect for an extended period of time [1,2]. Many drug delivery systems are based on so-called mucoadhesive polymers. Mucoadhesion is another version of bioadhesion, because the target is still the underlying tissue. These polymers are able to swell rapidly when placed in an aqueous environment and, therefore, exhibit a controlled drug release [3]. The interaction between the mucus and mucoadhesive polymers is a result of physical entanglement and secondary bonding, mainly $\mathrm{H}$-bonding and van der Waals attraction. These forces are related to the chemical structure of the polymers [4]. Typical polymers that have been used as mucoadhesive drug carriers include poly(acrylic acid) (PAA), poly-(methacrylic acid), carboxymethyl cellulose and hydroxypropyl methylcellulose [5-8]. The high water solubility of PAA critically limits its use as a TMD system to be applied in the buccal mucous membrane, because it may dissolve before the desired duration for the drug to permeate across the membrane [9].

The objectives of this study were to decrease the water solubility of PAA by preparing a PAA hydrogel by electron beam irradiation and to maintain its mucoadhesive property in a TMD system. Polymer hydrogels are defined as physically or chemically crosslinked polymer networks to absorb a large amount of water without being dissolved [10,11]. There are two methods to prepare hydrogels using hydrophilic polymers. One is the use of radiation for the hydrogels. The other is the use of chemicals for the crosslinking networks. It is well known for the latter that residual chemical to crosslinking affects living cells when the polymer is used as a biomaterial. In this experiment, the radiation process was tried to prepare a bioadhesive drug delivery system.

\section{Experimental Section}

\subsection{Materials}

Poly(acrylic acid) (PAA) with $M_{\mathrm{W}} 1,000,000$ was purchased from Waco Pure Chemical Industries, Ltd. (Osaka, Japan) and used without further purification. Triamcinolone acetonide (TAA) was supplied by Sigma Aldrich (St. Louis, MO, USA) and also used without further purification.

\subsection{Preparation of Hydrogels for Drug Carriers}

In this experiment, PAA was selected to prepare the bioadhesive hydrogel adhering to mucosal surfaces using a radiation process. PAA was dissolved in water to prepare 1, 3, 5, 7 and $9 \mathrm{wt} \%$ aqueous PAA solutions; the 3-mL PAA solution was put in a 35-mm petri dish and irradiated by an electron beam accelerator ( $10 \mathrm{MeV} / 1 \mathrm{~mA}$, Jeongup site of KAERI, Junbuk, Korea) at up to $75 \mathrm{kGy}$ to make the hydrogels. 


\subsection{Determination of Gelation}

The hydrogels prepared by irradiation were dried to measure the gelation. The dried hydrogels were extracted with water for $24 \mathrm{~h}$ at room temperature in order to extract the insoluble parts of the hydrogel. The insoluble part, i.e., the gelled part, was taken out and washed with water to remove the soluble part and then dried and weighed. This extraction cycle was repeated until the weight became constant. The gel percent in the hydrogel was determined from the following equation:

$$
\text { Degree of gelation }(\%)=\left(W_{e} / W_{d}\right) \times 100 \%
$$

where $W_{d}$ and $W_{e}$ represent the weights of the dry hydrogel and the gelled part after extraction, respectively.

\subsection{Determination of Swelling}

The dried hydrogels, which were punched into discs, were weighed and allowed to swell in distilled water. The degree of swelling at equilibrium swelling was calculated as follows:

$$
\text { Swelling percent }(\%)=\left[\left(W_{s}-W_{d}\right) / W_{d}\right] \times 100 \%
$$

where $W_{d}$ and $W_{s}$ represent the weights of dry and wet hydrogel, respectively.

\subsection{Mucoadhesive Strength}

A buccal mucose of pig was used to determine the mucoadhesive properties of the hydrophilic crosslinked poly(acrylic acid). Hydrogels with a 3-mm thickness were dried, cut into a circle with a $10-\mathrm{mm}$ diameter and then fixed to the cylindrical probe (10 $\mathrm{mm}$ in diameter) by double-sided adhesive tape (Figure 1). The buccal mucose specimen of a pig was cut into a $30 \mathrm{~mm} \times 30 \mathrm{~mm}$ size, and fixed to the surface of a stainless steel plate. Peel testing of the sample film was carried out using a texture analyzer (TA.XT2i, Stable Micro Systems Ltd., Godalming, UK) with a 50-N load cell equipped with a mucoadhesive holder. Figure 1 shows a schematic of the mucoadhesive test process. A test probe with dried film was then moved downward to make contact with the wet mucose surface. By using the texture analyzer, the maximum force required to separate the probe from the mucose (i.e., maximum detachment force; $F_{\max }$ ) is calculated directly using Texture Exponent 32 software (Texture Technologies Corp, Hamilton, MA, USA).

\subsection{Drug Release}

TAA was used as a model drug. The PAA film, which was dried from a hydrogel, was dipped in a $0.1 \mathrm{wt} \%$ TAA solution in ethanol and then dried at $25{ }^{\circ} \mathrm{C}$. The drug release was determined by putting it in a PBS (Phosphate buffered saline) ( $\mathrm{pH}$ 7.4) in the vial and shaking with an Orbital Shaker (Scientific Industries, INC., New York, NY, USA) at $100 \mathrm{rpm}$ at room temperature. The release of TAA was determined at different time intervals, and UV-Vis spectroscopy (BioTek Instruments, Inc., Winooski, VT, USA) was used to determine the concentration of triamcinolone acetonide at $238 \mathrm{~nm}$. 
Figure 1. Process of the mucoadhesive test by the texture analyzer with a mucoadhesive holder. (a) The probe with hydrated poly(acrylic acid) (PAA) film was moved downward. (b) Dried PAA film was attached to pig buccal mucosa. (c) The probe is withdrawn at a specified rate.

(a)

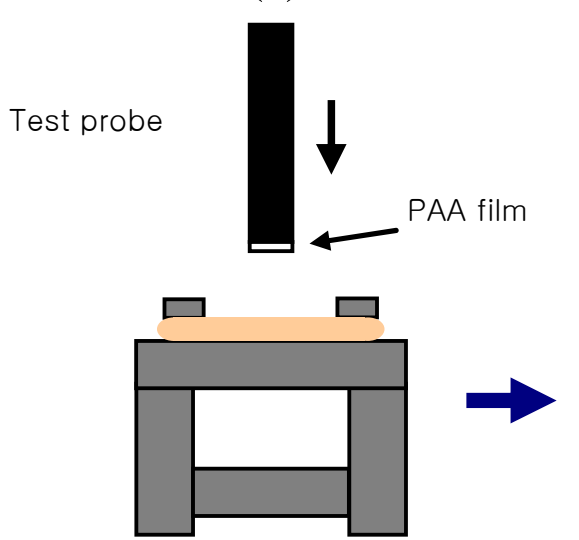

(b)

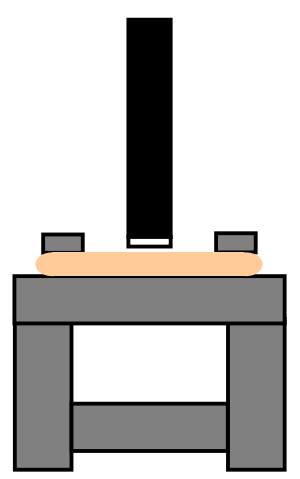

(c)

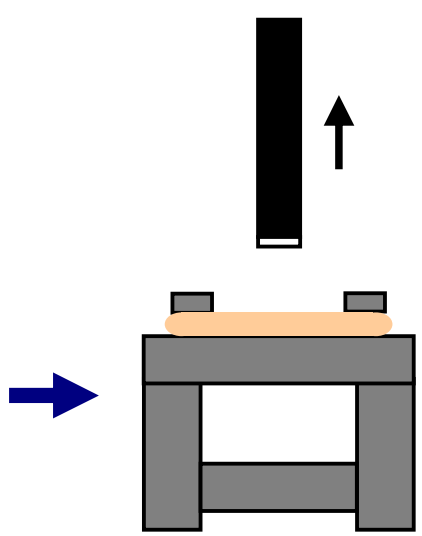

\section{Results and Discussion}

\subsection{Gel Percent and Swelling Behavior}

Thus far, a considerable number of studies have been performed on the crosslinking of PAA in aqueous solution by using irradiation [12-14]. Generally, the PAA hydrogels were crosslinked through free radical formation on the polymer chains during the exposure to irradiation. Furthermore, the radiolysis of water molecules generates the formation of hydroxyl groups, which can attack polymer chains, resulting in the formation of microradicals [15].

The effect of irradiation dose on the gel percent in various concentrations of PAA is shown in Figure 2. It was observed that the gel part increased with an increase in irradiation dose and the concentration of PAA. Crosslinking by radiation transforms a linear polymer into a three-dimensional molecule, resulting in a significant increase with a lower solubility in organic solvents. Therefore, it is explained that the increase in the gel fraction by irradiation and the concentration of PAA, as shown in Figure 2, is mostly due to the crosslinking of the polymer.

Figure 3 shows the swelling behavior of the dried PAA films in distilled water. In this experiment, the radiation dose was $50 \mathrm{kGy}$. It was observed that the polymer swelling decreased with an increase in the concentration of PAA. This is attributable to the three-dimensional network in water increasing with the concentration of PAA, resulting in a restriction in the mobility of the polymer chains. These lead to a decrement in the swelling of the hydrogel. 
Figure 2. The effect of irradiation dose on the gel content in various concentration of poly(acrylic acid).

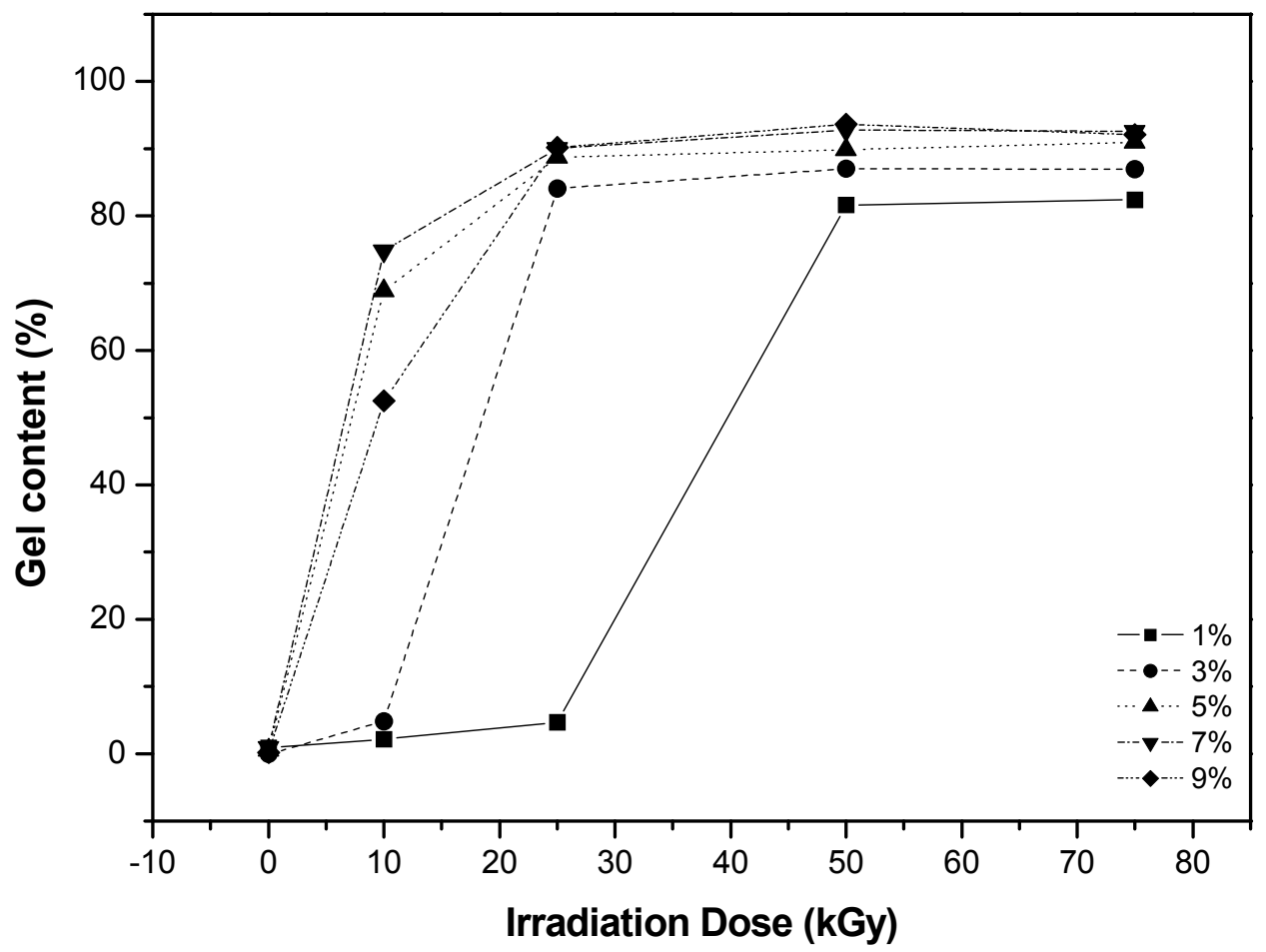

Figure 3. Effect of irradiation dose on the swell percent of the crosslinked poly(acrylic acid).

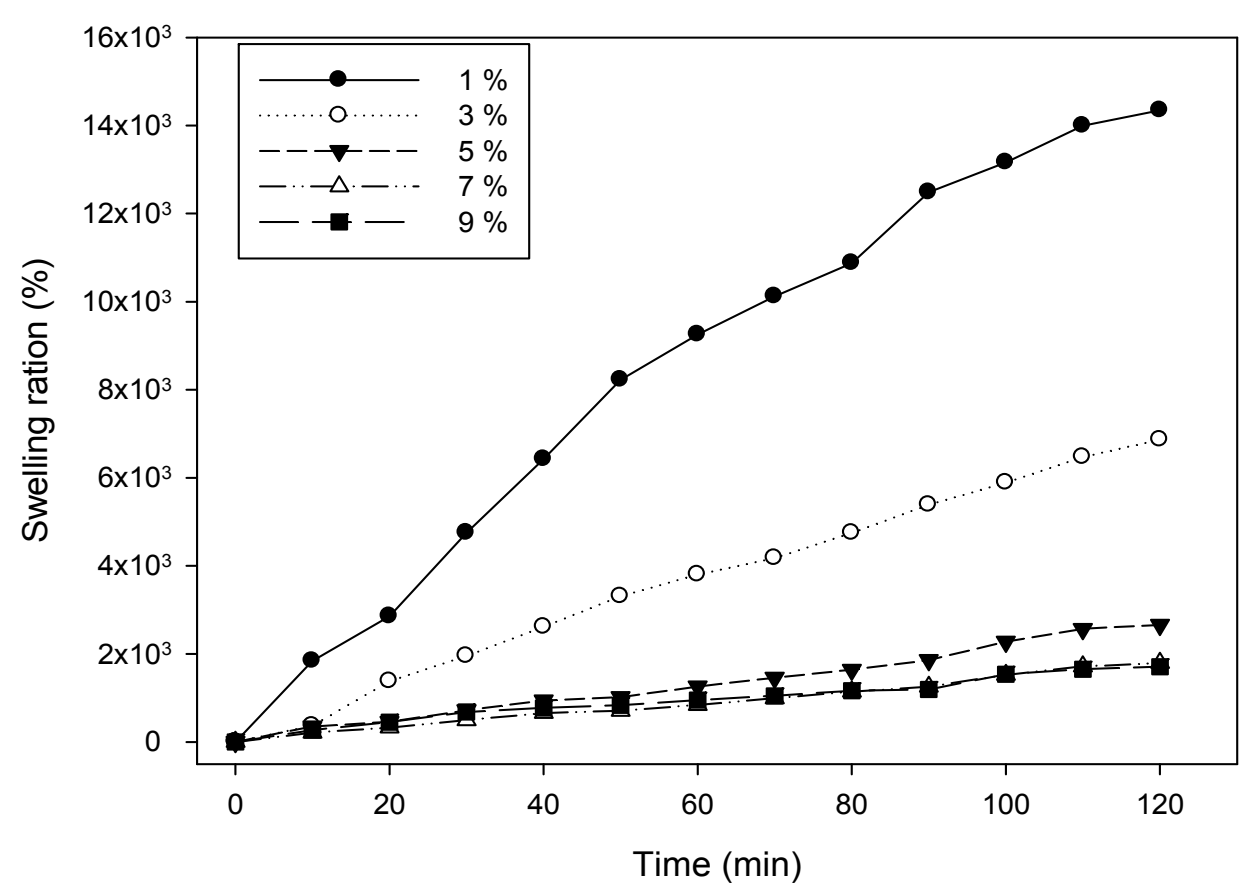

\subsection{Mucoadhesive Properties}

The mucoadhesive strength for the application of buccal mucosa is important to prolong the residence time of the drug. The buccal mucose from a pig was used to determine the mucoadhesive 
properties of the hydrophilic crosslinked PAA-based specimens. Hydrogels of $3 \mathrm{~mm}$ in thickness were dried, cut into $10 \mathrm{~mm}$ diameter circles and then fixed to a cylindrical probe (10 $\mathrm{mm}$ in diameter) using double-sided adhesive tape (Figure 1a ). A buccal mucose specimen of pig was cut into a $30 \mathrm{~mm} \times 30 \mathrm{~mm}$ size and fixed to the surface of a stainless steel plate. Peel testing of the sample film was carried out using a universal mechanical tester equipped with a mucoadhesive holder. The probe with a PAA-based polymer disc was pulled out at a speed of $0.5 \mathrm{~mm} / \mathrm{s}$ after attachment to the mucose surface at a contact force of $0.05 \mathrm{~N}$ and a contact time of $60 \mathrm{~s}$.

Shin et al., reported that the mucoadhesive strength of the pluronic F127 polymer was about $10 \mathrm{kPa}$ [16]. Figure 4 shows the average mucoadhesive strength between PAA films and the mucose surface as the contents of PAA increased from $1 \%$ to $9 \%$. In this experiment, the irradiation dose of PAA films was $50 \mathrm{kGy}$. The mucoadhesive strength decreased with an increase the concentration of PAA. As shown in Figure 4, $1 \mathrm{wt} \%$ PAA had a mucoadhesive strength, which was $62 \mathrm{kPa}$.

As a possible mechanism, the lower swelling ratio at a higher crosslinking resulted in a decrease in mucoadhesive strength. Generally, the mucoadhesion occurs in the mucosa environment; carboxyl groups interact with mucin molecules in their protonated form [17]. The role of the carboxyl group is the formation of hydrogen bonds with mucin molecules [16]. However, the mucoadhesive strength at a higher concentration of PAA was decreased despite the increased number of carboxyl groups. The probable reason for this result is that the chain flexibility of the polymer chain is decreased, due to the high crosslinking density. The chains of polymer having high flexibility may create more of the interfacial contact and provide a better environment for entanglement between the polymer and the mucin molecules [17].

Figure 4. Effect of irradiation dose on the mucoadhesive strength.

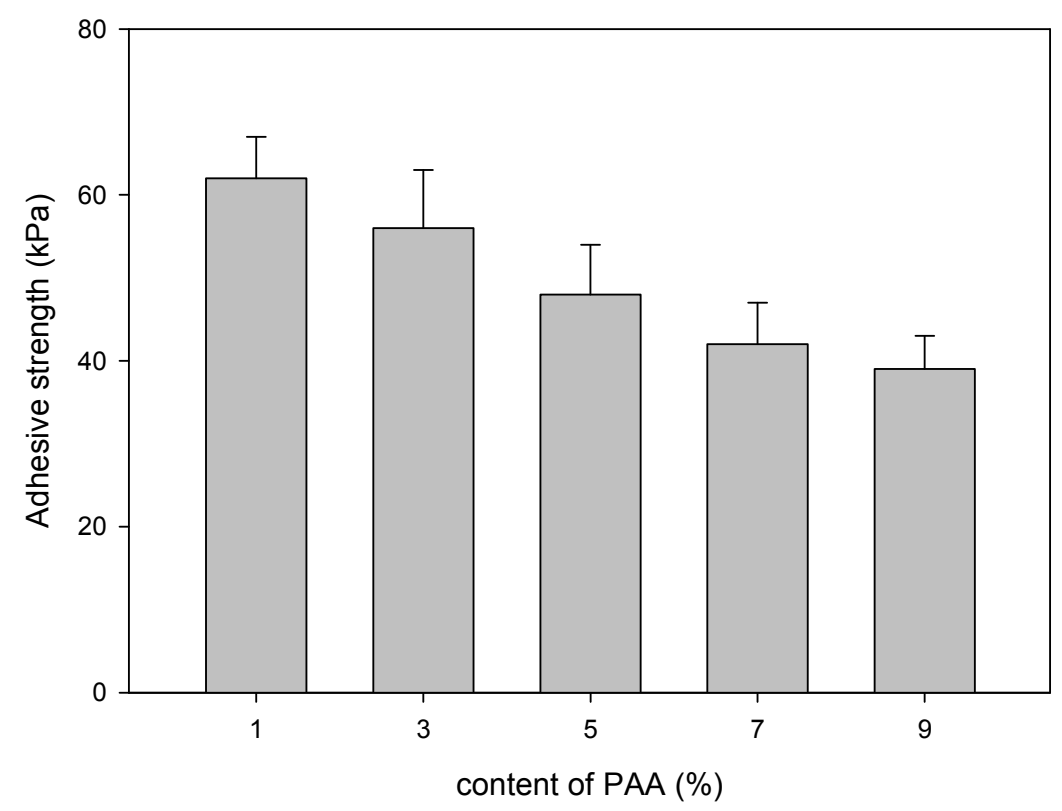

\subsection{Release Rate}

The dried PAA (content: $1 \mathrm{wt} \%$; irradiation dose: $50 \mathrm{kGy}$ ) film was dipped in a $0.1 \mathrm{wt} \%$ triamcinolone acetonide (TAA) solution in ethanol and then dried at $25{ }^{\circ} \mathrm{C}$. The drug release was 
determined by putting it in PBS ( $\mathrm{pH}$ 7.4). The release of TAA was determined at different time intervals by UV spectroscopy.

The TAA drug release profiles from the PAA hydrogel were investigated as shown in Figure 5. The drug was constantly released from the PAA hydrogel and could reach $90 \%$ at about 300 min in PBS. However, the release content was comparatively high at up to $60 \mathrm{~min}$ and then increased slightly. The probable reason for the TAA drug release from PAA hydrogel is that TAA is released by diffusion through the three-dimensional network structure of the PAA hydrogel, resulting in the initial burst $[16,18]$.

Figure 5. Drug release of crosslinked PAA loaded with triamcinolone acetonide. TAA, triamcinolone acetonide.

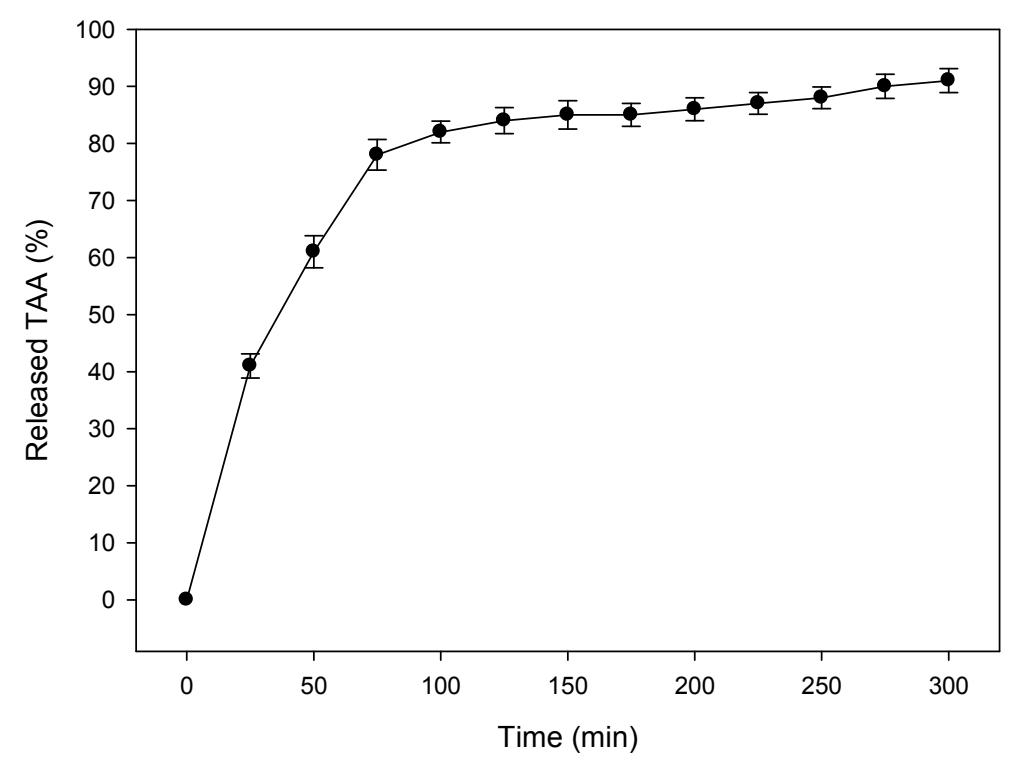

\section{Conclusions}

This paper describes a bioadhesive drug delivery system to be administered by adhering to the wet mucosa surface of a mucosa membrane. In this experiment, PAA was selected to prepare the bioadhesive specimen adhering to mucosal surfaces using a radiation process. An aqueous PAA solution was easily crosslinked by electron beam radiation. It was shown that poly(acrylic acid)-based drug carriers were successfully prepared for use in a bioadhesive drug delivery system.

\section{Acknowledgements}

This work was supported by National Research Foundation of Korea (NRF) grant funded by the Ministry of Science, ICT (Information \& Communication Technology) and Future Planning, Korea government (No.2013M2A2A6042482)

\section{Author Contributions}

Park and Nho contributed equally to this work. 


\section{Conflicts of Interest}

The authors declare no conflict of interest.

\section{References}

1. Florence, A.T.; Jani, P.U. Novel oral drug formulations. Their potential in modulating adverse-effects. Drug Saf. 1994, 410, 233-266.

2. Hwang, S.J.; Park, H.; Park, K. Gastric retentive drug-delivery systems. Crit. Rev. Ther. Drug Carrier Syst. 1998, 15, 243-284.

3. Choi, H.K.; Kim, O.J.; Chung, C.K.; Cho, C.S. A novel mucoadhesive polymer prepared by template polymerization of acrylic acid in the presence of poly(ethylene glycol). J. Appl. Polym. Sci. 1999, 73, 2749-2754.

4. Claudia, V. The use of mucoadhesive polymers in vaginal delivery. Adv. Drug Deliv. Rev. 2005, $57,1692-1712$.

5. Chun, M.K.; Cho, C.S.; Choi, J.K. Mucoadhesive drug carrier based on interpolymer complex of poly(vinyl pyrrolidone) and poly(acrylic acid) prepared by template polymerization. J. Control. Release 2002, 81, 327-334.

6. Abruzzo, A.; Bigucci, F.; Cerchiara, T.; Cruciani, F.; Vitali, B.; Luppi, B. Mucoadhesive chitosan/gelatin films for buccal delivery of propranolol hydrochloride. Carbohydr. Polym. 2012, $87,581-588$.

7. Cao, Q.R.; Liu, Y.; Xu, W.J.; Lee, B.J.; Yang, M.; Cui, J.H. Enhanced oral bioavailability of novel mucoadhesive pellets containing valsartan prepared by a dry powder-coating technique. Int. J. Pharm. 2012, 434, 325-333.

8. Juntapram, L.; Praphairaksit, N.; Puttipipatkhachorn, S.; Sriamornsak, P. Synthesis and characterization of chitosan-homocysteine thiolactone as a mucoadhesive polymer. Cabohydr. Polym. 2012, 87, 2399-2408.

9. Yang, X.; Robinson, J.R. Bioadhesion in mucosal drug delivery. In Biorelated Polymers and Gels; Okano, T., Ed., Academic Press: Sandiego, CA, USA, 1998; pp. 135-192.

10. Ian, G.N.; Frederick, C.S. In vitro assessment of bioadhesion for periodontal and buccal drug delivery. Biomaterials 1995, 16, 617-624.

11. Pastorczak, M.; Kadlubowski, S. Molecular relaxations in radiationally crosslinked poly(vinyl methyl ether) hydrogels. J. Non Cryst. Solids 2007, 353, 4536-4540.

12. Pappas, N.A.; Sahlin, J.J. Hydrogel as mucoadhesive and bioadhesive materials: A review. Biomaterials 1996, 17, 1553-1561.

13. Bell, C.L.; Pappas, N.A. Water, soluble and protein diffusion in physiologically responsive hydrogels of poly(methacrylic acid-g-ethyleneglycol). Biomaterials 1996, 17, 1203-1218.

14. Peppas, N.A.; Klier, J. Controlled release by using poly(methacrylic acid-g-ethyleneglycol) hydrogels. J. Control. Release 1991, 16, 203-214.

15. Jabbari, E.; Nozari, S. Synthesis of acrylic acid hydrogel by g-irradiation crosslinking of poly(acrylic acid) in aqueous solution. Iran Polym. J. 1999, 8, 263-270. 
16. Shin, B.K.; Baek, E.J.; Choi, S.G.; Davaa, E.; Nho, Y.C.; Lim, Y.C.; Park, J.S.; Huh, K.M.; Park, J.S. Preparation and irradiation of pluronic F127-based thermoreversible and mucoadhesive hydrogel for local delivery of naproxen. Drug Dev. Ind. Pharm. 2013, 39, 1874-1880.

17. Park, H.; Robinson, J.R. Mechanisms of mucoadhesion of poly(acrylic acid) hydrogels. Pharm. Res. 1987, 4, 457-464.

18. Choi, S.G.; Baek, E.J.; Davaa, E.; Nho, Y.C.; Lim, Y.C.; Park, J.S.; Gwon, H.J.; Huh, K.M.; Park, J.S. Topical treatment of the buccal mucosa and wounded skin in rats with a triamcinolone acetonide-loaded hydrogel prepared using an electron beam. Int. J. Pharm. 2013, 447, 102-108.

(C) 2014 by the authors; licensee MDPI, Basel, Switzerland. This article is an open access article distributed under the terms and conditions of the Creative Commons Attribution license (http://creativecommons.org/licenses/by/3.0/). 